\title{
Piezoelektrische Potentialfelder um Schraubenversetzungen beliebiger Ridhtung in piezoelektrischen Kristallen mit elastischer Isotropie
}

\author{
Von L. Merten \\ Philips Zentrallaboratorium GmbH, Aachen \\ (Z. Naturforschg. 19 a, 788-793 [1964] ; eingegangen am 10. Februar 1964)
}

\begin{abstract}
Versetzungen in piezoelektrischen Kristallen sind auf Grund des Piezoeffekts im allgemeinen von elektrischen Potentialen umgeben. Sie besitzen unter der Voraussetzung elastischer Isotropie eine sehr einfache und allgemeine Gestalt: Für Schraubenversetzungen, auf die sich die folgende Untersuchung speziell bezieht, ergibt sich für beliebige Richtungen der Versetzungslinien unter Ausschluß des Versetzungskerns ein nur winkelabhängiges Potential der Form $V=D \cos 2 \varphi$ (mit $D=$ const). Nur für wenige Richtungen hoher Symmetrie verschwinden die Potentiale auf Grund der Kristallsymmetrie $(D=0)$. Für piezoelektrische kubische Kristalle und hexagonale der Klasse $\mathrm{C}_{6 \mathrm{v}}$ werden diese Ausnahmerichtungen angegeben. $\mathrm{Zu}$ diesen Klassen gehören insbesondere die technisch wichtigen Kristalle mit Zinkblende- und Wurtzitstruktur.
\end{abstract}

\section{Einleitung}

In einer vorhergehenden Arbeit ${ }^{1}$ wurde gezeigt, daß Versetzungen in piezoelektrischen Kristallen im allgemeinen von einem durch den Piezoeffekt bedingten elektrischen Feld umgeben sind; den Versetzungen entlang verlaufen "Potentialwälle“ und „-gräben“. Wir nennen sie im folgenden auch kurz piezoelektrische Felder bzw. Potentiale der Versetzungen. Als Beispiel wurde in ${ }^{1}$ im einzelnen das piezoelektrische Feld und Potential $V$ um eine $\langle 001\rangle$-Schraubenversetzung berechnet, wobei sich (bei Abwesenheit wahrer Ladungen) ein Verlauf ${ }^{2}$ von $V$ nach [vgl. $(2,9)$ in ${ }^{1}$ ]

$$
V=D \cos 2 \varphi
$$

ergab, wenn man Polarkoordinaten $(r, \varphi)$ in einer Ebene senkrecht zur Schraubenachse benutzt.

Um bei experimentellen Untersuchungen möglichst eindeutig den Einfluß dieser piezoelektrischen Potentiale, z. B. auf elektrische Leitungsvorgänge und optische Prozesse im Kristall, von bekannten und denkbaren sonstigen Einflüssen von Versetzungen trennen zu können, ist die Beantwortung der Frage von Wichtigkeit, welchen Verlauf die piezoelektrischen Potentiale auch anderer Versetzungen zeigen und ob diese etwa gemeinsame Züge aufweisen. Dabei sind alle auf Grund der Stabilitätskriterien zu-

1 L. Merten, Phys. kondens. Materie 2, 53, 66 [1964]. In Gl. $(2,3)$ dieser Arbeit ist abzuändern: $\varrho \equiv r / r^{\prime}$ in $\varrho \equiv r^{\prime} / r$ und $R^{\prime}=r^{\prime} \sqrt{\varrho^{2}+\ldots}$ in $R^{\prime}=r \sqrt{\varrho^{2}+\ldots .}$.

2 In $(1,1)$ bleibt der Versetzungskern von der Betrachtung ausgeschlossen. Dies ist auch der Grund, weshalb in $(1,1)$ der Nullpunkt als eine „unphysikalische“ Singularität, gelassenen Richtungen einzuschließen. Um aber die Stabilitätsfragen hier nicht im einzelnen diskutieren zu müssen, werden wir im folgenden sogar beliebige Richtungen zulassen, so daß nachträglich für die jeweils betrachteten Kristalle noch zu untersuchen bleibt, für welche dieser Richtungen überhaupt stabile Versetzungen möglich sind. Dabei ist von besonderem Interesse, ob auch Ausnahmerichtungen mit identisch verschwindendem Potential existieren. Diese Fragen seien hier für gerade Schraubenversetzungen beantwortet, unter der Voraussetzung, daß sich der Kristall (nahezu) elastisch isotrop verhält. An anderer Stelle seien Stufenversetzungen unter der gleichen Voraussetzung behandelt.

Das Ergebnis ist überraschend einfach. Der Ausdruck $(1,1)$ stellt für elastisch isotrope Kristalle bereits den allgemeinsten Verlauf der piezoelektrischen Potentiale für Schraubenversetzungen dar. Die Abhängigkeit von der speziellen Richtung und der Symmetrie des piezoelektrischen Tensors der jeweils betrachteten Kristallklasse drücken sich lediglich in der Größe des für jede Versetzung konstanten Faktors $D$ aus. (Über den Nullpunkt von $\varphi$ hat man dabei natürlich passend zu verfügen.) Auch die Ausnahmerichtungen $(D=0)$ lassen sich aus der allgemeinen Darstellung des Faktors $D$ durch die Richtungskosinus und piezoelektrischen Konstanten für alle Kristallklassen relativ leicht ablesen. Wie gezeigt

nämlich eine Unstetigkeitsstelle, erscheint. Bezüglich einer näheren Diskussion der zu erwartenden Verhältnisse am Rande des Versetzungskerns und am äußeren Rande des Versetzungsgebiets (Versetzungshofes) sei auf ${ }^{1}$, insbesondere Teil I, Gl. (2,8), den Anhang und Teil II, Abschn. 5, verwiesen. 
wird, sind dies für piezoelektrische kubische Kristalle (fehlendes Symmetriezentrum: Klassen $\mathrm{T}$ und $\mathrm{T}_{\mathrm{d}}$ ), zu denen z. B. die technisch wichtigen Kristalle $\mathrm{ZnS}$, GaAs (Raumgruppe $\mathrm{T}_{\mathrm{d}}{ }^{2}$ ) gehören, nur die $\langle 111\rangle$ Richtungen und die $\langle 110\rangle$-Richtungen, für hexagonale Kristalle der Klasse $\mathrm{C}_{6 \mathrm{v}}$, zu denen z. B. die technisch ebenfalls wichtigen Kristalle CdS, CdSe, $\mathrm{ZnO}$ (Raumgruppe $\mathrm{C}_{6 \mathrm{v}}^{4}$ ) gehören, die Richtung der $c$-Achse und alle hierzu senkrechten Richtungen in der hexagonalen Basisebene.

\section{Darstellung der piezoelektrischen Potentiale um Schraubenversetzungen bei elastischer Isotropie}

Das Verzerrungsfeld einer geraden Schraubenversetzung in elastisch isotropen Kristallen wird dargestellt durch (vgl. Anm. ${ }^{3}$, S. 534, oder Anm. ${ }^{4}$, S. 115)

$$
\begin{gathered}
\varepsilon_{13}=\varepsilon_{31}=-\frac{b}{4 \pi} \frac{y}{r^{2}}, \\
\varepsilon_{23}=\varepsilon_{32}=\frac{b}{4 \pi} \frac{x}{r^{2}} .
\end{gathered}
$$

Hierin bedeutet $b$ den Betrag des Burgers-Vektors (mit Vorzeichen ${ }^{5}$ ), $x, y$ sind kartesische Koordinaten in der Ebene senkrecht zur Versetzungslinie ( $z$-Achse), die Indizes 1, 2, 3 beziehen sich wie üblich der Reihe nach auf die $x-, y$-, $z$-Achse.

Die zugehörige Dichte $\varrho^{\prime}$ der Polarisationsladungen wird allgemein gegeben durch [vgl. $\left.{ }^{1}, \mathrm{Gl} .(1,4)\right]$

$$
\varrho^{\prime}=-\sum_{\alpha} \sum_{\beta \gamma} e_{\alpha, \beta \gamma}^{\prime} \frac{\partial}{\partial x_{\alpha}} \varepsilon_{\beta \gamma}, \quad(\alpha, \beta, \gamma=1,2,3),
$$

worin die $e_{\alpha, \beta}^{\prime}$, die Komponenten des piezoelektrischen Tensors bedeuten. Das gesuchte Potentialfeld ergibt sich schließlich aus der mit diesem $\varrho^{\prime}$ gebildeten Poisson-Gl. (bei Abwesenheit wahrer Ladungen, insbesondere also von Elektronen und Löchern):

$$
\Delta V=-(4 \pi / \varkappa) \varrho^{\prime},
$$

worin $\approx$ die dielektrische Konstante ${ }^{6}$ bedeutet. Die

3 A. SEeger, Theorie der Gitterfehlstellen, im Handbuch der Physik, Bd. VII, Teil I., Verlag Springer, Berlin-Göttingen-Heidelberg 1955.

4 W. T. Read, Dislocations in Crystals, McGraw-Hill, New York-Toronto-London 1953. - Zur Umrechnung von den Schubspannungen auf die Scherungen sind die Gln. $(8,2)$ und $(8,3)$ noch mit $2 G=2 \mu$ zu dividieren.

${ }^{5} \mathrm{Im}$ Gegensatz zu ${ }^{3}$ und ${ }^{1}$ bedeutet positives $b$ hier eine Rechtsschraube, negatives eine Linksschraube. piezoelektrischen Konstanten $e_{\alpha, \beta \gamma}^{\prime}$ in $(2,2)$ sind bei Benutzung der Ausdrücke $(2,1)$ ebenfalls auf das der Versetzung angepaßte Koordinatensystem zu beziehen, außerdem ist dann natürlich zu setzen: $x_{1}=x, x_{2}=y, x_{3}=z$. Da mit diesen $e_{\alpha, \beta \gamma}^{\prime}$ sich die folgenden allgemeinen Ergebnisse direkt gewinnen lassen, ist es zweckmäßig, letztere erst nachträglich (Abschn. 3) durch die auf die jeweiligen Kristallachsen bezogenen $e_{\alpha, \beta}$, auszudrücken. Beim Einsetzen von $(2,1)$ sieht man nun sofort, daß in $(2,2)$ höchstens vier Glieder auftreten, nämlich diejenigen, deren zugehörige piezoelektrische Konstanten die hinteren Indexpaare 13 (bzw. 31) und 23 (bzw. 32) enthalten, also: $e_{1,13}^{\prime}, e_{1,23}^{\prime}, e_{2,13}^{\prime}, e_{2,23}^{\prime}$. Wie für alle geraden Versetzungen scheiden dabei Glieder mit dem Index 3 als ersten Index der piezoelektrischen Konstanten aus, da alle Ausdrücke von der Koordinate $z$, nach der abgeleitet wird, unabhängig sind. Beachtet man noch, daß

$$
\begin{aligned}
& -\frac{\partial}{\partial x}\left(\frac{x}{x^{2}+y^{2}}\right)=\frac{\partial}{\partial y}\left(\frac{y}{x^{2}+y^{2}}\right)=\frac{x^{2}-y^{2}}{r^{4}}=\frac{\cos 2 \varphi^{\prime}}{r^{2}}, \\
& -\frac{\partial}{\partial x}\left(\frac{y}{x^{2}+y^{2}}\right)=-\frac{\partial}{\partial y}\left(\frac{x}{x^{2}+y^{2}}\right)=\frac{2 x y}{r^{4}}=\frac{\sin 2 \varphi^{\prime}}{r^{2}},
\end{aligned}
$$

so folgt also

$$
\varrho^{\prime}=d_{1} \frac{\cos 2 \varphi^{\prime}}{r^{2}}+d_{2} \frac{\sin 2 \varphi^{\prime}}{r^{2}}
$$

mit

$$
\begin{aligned}
& d_{1} \equiv \frac{b}{2 \pi}\left(e_{1,23}^{\prime}+e_{2,13}^{\prime}\right), \\
& d_{2} \equiv \frac{b}{2 \pi}\left(e_{2,23}^{\prime}-e_{1,13}^{\prime}\right) .
\end{aligned}
$$

Führt man jetzt

$$
\begin{aligned}
d & \equiv \sqrt{d_{1}{ }^{2}+d_{2}{ }^{2}} \\
& =\frac{b}{2 \pi} \sqrt{\left(e_{1,23}^{\prime}+e_{2,13}^{\prime}\right)^{2}+\left(e_{2,23}^{\prime}-e_{1,13}^{\prime}\right)^{2}}
\end{aligned}
$$

und $\varphi \equiv \varphi^{\prime}-\varphi_{0}$ ein, mit Definition von $\varphi_{0}$ nach

$$
\cos 2 \varphi_{0} \equiv d_{1} / d, \quad \sin 2 \varphi_{0} \equiv d_{2} / d,
$$

so erhält man den einfachen Ausdruck

$$
\varrho^{\prime}=d \frac{\cos 2 \varphi}{r^{2}} \text {. }
$$

${ }^{6}$ In Gl. (2,3) ist natürlich die zusätzliche Voraussetzung der dielektrischen Isotropie (skalare Dielektrizitätskonstante) enthalten. Da aber diese Voraussetzung für alle kubischen Kristalle bekanntlich streng erfüllt ist und für die genannten hexagonalen Kristalle ebenfalls hinreichend gut, bedeutet dies hier keine wesentliche Einschränkung der Allgemeinheit. Auch werden sich die Ergebnisse ohne größere Schwierigkeiten auf Kristalle mit ausgeprägter dielektrischer Anisotropie erweitern lassen. 
Hiermit ergibt sich schließlich für das Potential $V$ im ganzen Versetzungsgebiet, wenn man den Versetzungskern wieder von der Betrachtung ausschließt und auch die Einflüsse des äußeren Randes vernachlässigt, aus der Poisson-Gl. (2.3) :

$$
V=D \cos 2 \varphi \quad \text { mit } \quad D=\frac{\pi}{\varkappa} d .
$$

Wir haben also das Ergebnis: Der unter der Voraussetzung der elastischen (und dielektrischen) Isotropie allgemeinste Ausdruck für das Potential $V$ um gerade Schraubenversetzungen ist von der Form $(1,1)$. Daher gelten unter dieser Voraussetzung auch alle z. B. in ${ }^{1}$ gezogenen weiteren Schlüsse ${ }^{7}$ für gerade Schraubenversetzungen beliebiger Richtung in beliebigen (piezoelektrischen) Kristallen.

\section{Darstellung des Faktors $D$ für piezoelektrische kubische und hexagonale Kristalle der Klasse $\mathrm{C}_{6 \mathrm{v}}$ mit Angabe der Ausnahmerichtungen}

Um $D$ (bzw. $d$ ) in Abhängigkeit von der Richtung darzustellen, haben wir jetzt die vier, auf die Versetzungsrichtungen bezogenen piezoelektrischen Konstanten $e_{a, \beta \gamma}^{\prime}$ in $(2,5)$ bzw. $(2,6)$ durch die gewöhnlich benutzten, auf die Kristallachsen bezogenen Konstanten $e_{\alpha, \beta}$, auszudrücken. Bedeuten

$$
\begin{gathered}
a_{31}=\cos \alpha, \quad a_{32}=\cos \beta, \quad a_{33}=\cos \gamma \\
\left(\text { mit } \cos ^{2} \alpha+\cos ^{2} \beta+\cos ^{2} \gamma=1\right)
\end{gathered}
$$

die Richtungskosinus der Schraubenachse ( $z$-Achse) in bezug auf die Kristallachsen $\left(x_{1}, x_{2}, x_{3}\right)$, so wird die Koordinatentransformation vermittelt durch den Tensor

$$
A=\left(a_{\alpha \beta}\right)=\left(\begin{array}{ccc}
-\cos \beta / n & , \quad \cos \alpha / n \quad & 0 \\
-\cos \alpha \cos \gamma / n, & -\cos \beta \cos \gamma / n, & \left(\cos ^{2} \alpha+\cos ^{2} \beta\right) / n \\
\cos \alpha & , & \cos \beta \quad,
\end{array}\right)
$$

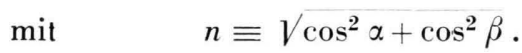

Die $x$-Achse wurde dabei auf die Schnittgerade der Ebene senkrecht zur $z$-Achse mit der $x_{1} x_{2}$-Ebene gelegt. - Als Komponenten eines Tensors dritter Stufe transformieren sich die piezoelektrischen Konstanten

$$
\begin{aligned}
& \text { nach [vgl. z. B. }{ }^{8}, \text { S. 111, Gl. (5) ] } \\
& \qquad e_{a, \beta \gamma}^{\prime}=\sum_{\lambda \mu \nu} a_{a \lambda} a_{\beta, \mu} a_{\gamma \nu} e_{\lambda, \mu \nu} .
\end{aligned}
$$

Für kubische Kristalle und weiter unten für Kristalle der hexagonalen Klasse $\mathrm{C}_{6 \mathrm{v}}$ seien die sich ergebenden Ausdrücke jetzt explizit angegeben.

Da in kubischen Kristallen nur

$$
e_{1,23}=e_{1,32}=e_{2,31}=e_{2,13}=e_{3,12}=e_{3,21}\left(\equiv e_{14}\right)
$$

von Null verschieden sind, treten in $(3,2)$ jeweils höchstens sechs Summanden auf (alle Kombinationen der Ziffern 123 als hintere Indizes der je drei Faktoren $a_{\alpha \beta}$ ), wobei von vornherein diejenigen fortgelassen werden können, in denen $a_{13}$ enthalten ist:

$$
\begin{aligned}
& \left.\begin{array}{l}
e_{1,23}^{\prime} \\
e_{2,13}^{\prime}
\end{array}\right\}=\frac{1}{n^{2}}\left(\cos ^{2} \alpha-\cos ^{2} \beta\right)\left(\cos ^{2} \alpha+\cos ^{2} \beta-\cos ^{2} \gamma\right) e_{14}, \\
& e^{\prime}{ }_{1,13}=-\frac{2}{n^{2}} \cos \alpha \cos \beta \cos \gamma e_{14}, \\
& e_{2,23}^{\prime}=-\frac{2}{n^{2}} \cos \alpha \cos \beta \cos \gamma\left(2 \cos ^{2} \alpha+2 \cos ^{2} \beta-\cos ^{2} \gamma\right) e_{14} .
\end{aligned}
$$

Unter Beachtung von $(3,2)$ folgt daher mit $l \equiv 2 e_{14} / n^{2}$ :

$$
\begin{aligned}
& e_{1,23}^{\prime}+e_{2,13}^{\prime}=l\left(\cos ^{2} \alpha-\cos ^{2} \beta\right)\left(2 \cos ^{2} \alpha+2 \cos ^{2} \beta-1\right), \\
& e_{2,23}^{\prime}-e_{1,13}^{\prime}=-l(\cos \alpha \cos \beta \cos \gamma)\left(3 \cos ^{2} \alpha+3 \cos ^{2} \beta-2\right) .
\end{aligned}
$$

${ }^{7}$ Sofern in ${ }^{1}$ zusätzliche Voraussetzungen gestellt sind (z. B. in Teil II : $e V<k T$ ), so sind diese natürlich jeweils erneut auf ihre Gültigkeit zu prüfen.
${ }^{8}$ J. F. Nye, Physical Properties of Crystals, At the Clarendon Press, Oxford 1957. 
Diese Formeln in Verbindung mit $(2,8)$ gestatten, die piezoelektrischen Potentiale bei Kenntnis von $b$, $\varkappa, e_{14}$ für jede vorgegebene Richtung zu berechnen. Insbesondere interessieren, wie erwähnt, die Ausnahmerichtungen mit $d=D=0$. Solche Richtungen liegen offensichtlich genau dann vor, wenn $(3,5 \mathrm{a})$ und $(3,5 b)$ gleichzeitig verschwinden, d. h. wenn verschwinden

x) der erste Klammerfaktor in (3,5a) und der erste in $(3,5 \mathrm{~b})^{9}$ :

$\cos \alpha= \pm 1 / \sqrt{2}, \quad \cos \beta= \pm 1 / \sqrt{2}, \quad \cos \gamma=0 ;$

$\beta$ ) der erste Faktor in $(3,5 \mathrm{a})$ und der zweite in $(3,5 b)$ :

$\cos \alpha= \pm 1 / \sqrt{3}, \cos \beta= \pm 1 / \sqrt{3}, \cos \gamma= \pm 1 / \sqrt{3} ;$

$\gamma)$ der zweite Faktor in $(3,5 \mathrm{a})$ und der erste in $(3,5 b)$ :

$\cos \alpha= \pm 1 / \sqrt{2}, \cos \beta=0, \quad \cos \gamma= \pm 1 / \sqrt{2} ;$ $\cos \alpha=0, \quad \cos \beta= \pm 1 / \sqrt{2}, \cos \gamma= \pm 1 / \sqrt{2}$;

$\delta)$ der zweite Faktor in $(3,5 a)$ und der zweite in $(3,5 b)$ :

Beide Bedingungen sind gleichzeitig nicht erfüllbar.

Die Fälle lassen sich zusammenfassen in dem Ergebnis: Unter den geraden Schraubenversetzungen in piezoelektrischen Kristallen mit kubischer Symmetrie sind frei von piezoelektrischen Potentialen nur etwaige $\langle 111\rangle$-Versetzungen $($ Fall $\beta$ ) und $\langle 110\rangle$-Versetzungen (Fall $\alpha+$ Fall $\gamma$ ). Wie nachträglich in einem Anhang gezeigt wird, ist dieses Ergebnis nicht an die Voraussetzung der elastischen Isotropie gebunden, gilt also allgemein.

Zweitens seien jetzt hexagonale Kristalle der Klasse $\mathrm{C}_{3 \mathrm{v}}$ behandelt. Bei diesen existieren drei voneinander unabhängige piezoelektrische Konstanten (vgl. z. B. ${ }^{8}$, S. 300; in Klammern in Voigtscher Indizierung) :

$$
\begin{aligned}
& e_{3,33}\left(\equiv e_{33}\right), e_{3,11}=e_{3,22}\left(\equiv e_{31}\right), \\
& e_{1,13}=e_{1,31}=e_{2,23}=e_{2,32}\left(\equiv e_{15}\right) .
\end{aligned}
$$

${ }^{9}$ Für $\cos \alpha=0, \cos \beta=0, \cos \gamma=1$, d. h. für die $\langle 001\rangle$-Richtungen, sind die vier $e_{\alpha, \beta \gamma}^{\prime}$ in $(3,4)$ nicht mehr eindeutig definiert, da die Grenzwerte

$\lim _{\left.\begin{array}{c}\cos \alpha \\ \cos \beta\end{array}\right\} \rightarrow 0} \frac{\cos ^{2} \alpha-\cos ^{2} \beta}{n^{2}}=\frac{0}{0} \quad$ und $\lim _{\begin{array}{c}\cos \alpha \\ \cos \beta\}\end{array}} \frac{\cos \alpha \cos \beta}{n^{2}}=\frac{0}{0}$

jetzt davon abhängen, aus welcher Richtung kommend man die $z$-Achse in die $x_{3}$-Achse übergehen läßt. Bei aufeinanderfallender $z$ - und $x_{3}$-Achse ist ja auch die $x$-Achse als Schnittgerade der jetzt ebenfalls zusammenfallenden $x y$ und $x_{1} x_{0}$-Ebenen gar nicht mehr eindeutig definiert. Dies ist jedoch nicht wesentlich, da
$\mathrm{Zu}$ dieser Klasse gehören z. B. die Kristalle mit Wurtzitstruktur, darunter die erwähnten, technisch wichtigen Kristalle $\mathrm{CdS}, \mathrm{CdSe}, \mathrm{ZnO}$, für die auch die Voraussetzung der elastischen Isotropie nach ${ }^{10,11}$ und ${ }^{12}$ relativ gut zutrifft.

Mit dem Schema $(3,1)$ ergibt sich wieder nach $(3,2)$ :

$$
\begin{aligned}
e_{1,23}^{\prime}= & 0, \\
e_{2,13}^{\prime}= & 0, \\
e_{1,13}^{\prime}= & \cos \gamma e_{15}, \\
e_{2,23}^{\prime}= & \left(\cos ^{2} \alpha+\cos ^{2} \beta\right) \cos \gamma e_{33} \\
& \quad-\left(\cos ^{2} \alpha+\cos ^{2} \beta\right) \cos \gamma e_{31} \\
& \quad+\left(1-2 \cos ^{2} \alpha-2 \cos ^{2} \beta\right) \cos \gamma e_{15}, \\
\text { also } \quad e_{1,23}^{\prime}+e_{2,13}^{\prime}=0, & \\
e_{2,23}^{\prime}-e_{1,13}^{\prime}= & \left(\cos ^{2} \alpha+\cos ^{2} \beta\right) \cos \gamma \\
& \quad \cdot\left[e_{33}-e_{31}-2 e_{15}\right] .
\end{aligned}
$$

Ausnahmerichtungen $(D=0)$ liegen wieder dann und nur dann vor, wenn $(3,9 \mathrm{~b})$ verschwindet, d. h. wenn

a) $\cos ^{2} \alpha+\cos ^{2} \beta=0$, also für die Richtung

$$
\cos \alpha=0, \quad \cos \beta=0, \quad \cos \gamma=1 .
$$

$\beta$ ) $\cos \gamma=0$, also für alle Richtungen mit

$$
\cos ^{2} \alpha+\cos ^{2} \beta=1, \quad \cos \gamma=0 .
$$

Beide Fälle lassen sich wieder zusammenfassen in dem Ergebnis: Unter den geraden Schraubenversetzungen in Kristallen der hexagonalen Klasse $\mathrm{C}_{6 \mathrm{v}}$ sind frei von piezoelektrischen Potentialen nur etwaige Versetzungen in Richtung der $c$-Achse (Fall $\alpha)$ und in allen hierzu senkrechten Richtungen in der hexagonalen Basisebene (Fall $\beta$ ). Wie im Anhang gezeigt wird, ist dieses Ergebnis ebenfalls nicht an die Voraussetzung der elastischen Isotropie gebunden.

Hier sei nochmals betont, daß bei der Angabe der Ausnahmerichtungen wieder unberücksichtigt blieb, ob die Schraubenversetzungen in den angege-

$$
\begin{aligned}
& \left(e^{\prime}{ }_{1,23}+e^{\prime} 2,13\right)^{2}+\left(e^{\prime} 2,23-e^{\prime} 1,13\right)^{2} \\
\rightarrow & l^{2}\left(\cos ^{2} \alpha-\cos ^{2} \beta\right)^{2}+4 l^{2} \cos ^{2} \alpha \cos ^{2} \beta \\
= & l^{2}\left(\cos ^{2} \alpha+\cos ^{2} \beta\right)^{2}=4 e^{2}{ }_{14},
\end{aligned}
$$

also auch $d=b e_{14} / \pi \neq 0$ [vgl. Gl. (2,6a) ] eindeutig bleiben. Die $\langle 001\rangle$-Richtungen, die im übrigen ja bereits in ${ }^{1}$ ausführlich behandelt wurden, sind also keine Ausnahmerichtungen.

10 E. Gutsche, Naturwiss. 23, 66 [1958] ; phys. stat. sol. 1, 30 [1961].

11 T. B. Bateman, J. Appl. Phys. 33, 3309 [1962].

12 D. Berlincourt, H. Jaffe u. L. R. Shrozawa, Phys. Rev. 129, 1009 [1963]. 
benen Richtungen tatsächlich stabil sind; dies ist jeweils noch nachträglich zu diskutieren. So sollten z. B. reine Schraubenversetzungen in den $\langle 111\rangle$ Ausnahmerichtungen der erwähnten Kristalle mit Zinkblendestruktur (ZnS, GaAs) nach den Stabilitätskriterien instabil sein (vgl. ${ }^{3}$, S. 485).

Für alle (fast elastisch isotropen piezoelektrischen) Kristalle der behandelten Kristallklassen lassen sich jetzt auch die numerischen Werte der piezoelektrischen Potentiale [nach $(2,8)$ mit $(2,6 a)$ und $(3,5)$ bzw. (3,9)] zu jeder Schraubenversetzung mit bekanntem Burgers-Vektor direkt angeben, falls die jeweiligen piezoelektrischen, elastischen und dielektrischen Konstanten bekannt sind. Dies gilt auch für Versetzungen mit gemischtem Charakter bezüglich des Schraubenanteils, da sich wegen der benutzten linearen Theorie natürlich auch die elektrischen $\mathrm{Po}_{0}$ tentiale des Schrauben- und Stufenanteils superponieren. Unter $b$ hat man dann den Betrag der Komponente des Burgers-Vektors in Richtung der Schraubenachse zu verstehen.

Da jedoch bisher für keine piezoelektrische Verbindung ${ }^{13}$ systematische Untersuchungen über die Art und Richtung der tatsächlich auftretenden Versetzungen vorzuliegen scheinen, sei auf eine numerische Berechnung an dieser Stelle verzichtet. Welche Größenordnungen man für einige der angeführten Verbindungen etwa zu erwarten hat, ergibt sich aus dem Vergleich mit den in ${ }^{1}$ und ${ }^{14}$ berechneten Werten der Potentiale einiger Versetzungen in Richtungen hoher Symmetrie.

Mehreren Kollegen, besonders Herrn Dr. J. Hornstra, möchte ich für wertvolle Hinweise und anregende Diskussionen sehr danken.

\section{Anhang}

Es wird gezeigt:

Für alle unter der Voraussetzung der elastischen Isotropie erhaltenen Ausnahmerichtungen $(V=0)$ für gerade Schraubenversetzungen bleibt der Charakter einer Ausnahmerichtung erhalten, auch wenn der Theorie die volle Symmetrie der elastischen Eigenschaften zugrunde gelegt wird.

13 Mit Ausnahme vielleicht von $\mathrm{InSb}$ und $\mathrm{GaAs}$, wobei aber für InSb auch der Wert der piezoelektrischen Konstante noch nicht bekannt ist.

14 L. Merten, Z. Naturforschg. 19 a [1964], im Druck.

15 J. D. Eshelby, Proc. Phys. Soc., Lond. A 62, 307 [1949].

16 J. D. Eshelby, W. T. Read u. W. Shockley, Acta Met. 1, 251 [1953].

17 A. Seeger u. G. Sснӧск, Acta Met. 1, 519 [1953].
Zur Berechnung der piezoelektrischen Potentiale der Versetzungen unter Berücksichtigung der vollen Symmetrie der Kristalle hat man wieder vom Verzerrungsfeld auszugehen. Dazu greifen wir zurück auf die elastische Theorie gerader Versetzungen in anisotropen Kristallen, die von Eshelby ${ }^{15}$, Eshelby, Read und ShockLey ${ }^{16}$, Seeger und Schöck ${ }^{17}$, Foreman ${ }^{18}$ u. a. ausgebaut wurde. Wie ausführlich von $\mathrm{CHOU}^{19}$ gezeigt wurde, erhält man für das Spannungsfeld (und folglich auch für das Verzerrungsfeld) einfache analytische Ausdrücke derselben Form, wenn die Versetzungsrichtung folgende Bedingungen erfüllt: Die Versetzungsrichtung ( $z$-Richtung) steht senkrecht auf einer Symmetrieebene ${ }^{20}$ und liegt selbst in einer Symmetrieebene ( $x z$-Ebene). Wie man sich leicht überzeugt, trifft dies für alle im Hauptteil angegebenen Ausnahmerichtungen zu, wenn man von den Richtungen in der hexagonalen Basisebene (=xz-Ebene) zunächst nur diejenigen entlang der $a$ Achsen und senkrecht dazu einbezieht.

Für diese Richtungen vereinfacht sich die Matrix der elastischen Konstanten zu

$$
C \equiv\left(\begin{array}{llllll}
c^{\prime}{ }_{11} & c^{\prime}{ }_{12} & c^{\prime}{ }_{13} & 0 & 0 & 0 \\
c^{\prime}{ }_{12} & c^{\prime}{ }_{22} & c^{\prime}{ }_{23} & 0 & 0 & 0 \\
c^{\prime}{ }_{13} & c^{\prime}{ }_{23} & c^{\prime}{ }_{33} & 0 & 0 & 0 \\
0 & 0 & 0 & c^{\prime}{ }_{44} & 0 & 0 \\
0 & 0 & 0 & 0 & c^{\prime}{ }_{85} & 0 \\
0 & 0 & 0 & 0 & 0 & c_{66}^{\prime}
\end{array}\right) .
$$

Da sich aber diese Matrix bei einer Drehung der $z$-Achse [es bestehen jetzt zusätzlich die Beziehungen: $c^{\prime}{ }_{11}=c^{\prime}{ }_{33}$ $=c_{11}=c_{22}, \quad c_{12}^{\prime}=c^{\prime}{ }_{23}=c_{13}=c_{23}, \quad c^{\prime}{ }_{44}=c_{66}^{\prime}=c_{44}=c_{55}$, $\left.c^{\prime}{ }_{55}=\frac{1}{2}\left(c^{\prime}{ }_{11}-c^{\prime}{ }_{13}\right)=\frac{1}{2}\left(c_{11}-c_{12}\right)\right]$ in der hexagonalen Basisebene nicht ändert, gilt also $(\mathrm{A}, 1)$ für alle Versetzungsrichtungen in der hexagonalen Basisebene. Für Schraubenversetzungen treten jetzt wieder nur Schubspannungen auf, die nach ${ }^{19}$ die Form besitzen:

$$
\begin{aligned}
\sigma_{13} & =-\frac{K b}{2 \pi} \frac{\eta^{2} y}{x^{2}+\eta^{2} y^{2}}, \\
\sigma_{23} & =\frac{K b}{2 \pi} \frac{x^{2}}{x^{2}+\eta^{2} y^{2}}
\end{aligned}
$$

mit

$$
K \equiv \sqrt{c_{44}^{\prime} c_{55}^{\prime}}, \quad \eta \equiv \sqrt{c_{55}^{\prime} / c^{\prime}{ }_{44}} \text {. }
$$

Aus (A,1) und (A,2) ergibt sich dann sofort für die Komponenten des Verzerrungstensors:

$$
\begin{aligned}
& \varepsilon_{13}=\frac{\sigma_{13}}{2 c^{\prime}{ }_{55}}=-\frac{b \eta}{4 \pi} \frac{y}{x^{2}+\eta^{2} y^{2}}, \\
& \varepsilon_{23}=\frac{\sigma_{23}}{2 c^{\prime}{ }_{44}}=\frac{b \eta}{4 \pi} \frac{x}{x^{2}+\eta^{2} y^{2}} .
\end{aligned}
$$

Dabei soll der obere Strich an den elastischen Konstanten wieder darauf hindeuten, daß die elastischen Konstanten ebenfalls auf das der Versetzung angepaßte

18 A. J. E. Foreman, Acta Met. 3, 322 [1955].

19 Y. T. Chоv, J. Appl. Phys. 34, 429 [1963].

20 Bei der Betrachtung der elastischen Symmetrie stört bekanntlich nicht, daß z. B. in den Kristallen der Klasse $\mathrm{C}_{6 \mathrm{v}}$ die Gitterebenen senkrecht zur $c$-Achse zwar Symmetrieebenen für die Teilgitter, aber nicht für das zusammengesetzte Gitter sind, d.h. daß die $c$-Achse (=z-Achse) in Wirklichkeit eine polare Achse ist. 
Koordinatensystem zu beziehen sind. Die expliziten Beziehungen dieser $c^{\prime}{ }_{\alpha \beta}$ mit den auf die Kristallachsen bezogenen $c_{\alpha \beta}$ werden jedoch für das Folgende nicht benötigt. Für die spätere Diskussion ist lediglich zu bemerken, daß z. B. für die Versetzungen entlang einer 〈111〉-Richtung der kubischen Kristalle oder entlang der $c$-Achse der hexagonalen Kristalle $c^{\prime}{ }_{55}=c^{\prime}{ }_{44}$ und folglich $\eta=1$ wird, so daß die Ausdrücke (A,3) für die Verzerrungskomponenten dann wieder mit $(2,1)$ übereinstimmen.

Beachtet man, daß

$$
\begin{aligned}
-\frac{\partial}{\partial x}\left(\frac{y}{x^{2}+\eta^{2} y^{2}}\right) & =\frac{1}{\eta} \frac{2 x \eta y}{\left(x^{2}+\eta^{2} y^{2}\right)^{2}}, \\
-\frac{\partial}{\partial x}\left(\frac{x}{x^{2}+\eta^{2} y^{2}}\right) & =\frac{\partial}{\partial y}\left(\frac{y}{x^{2}+\eta^{2} y^{2}}\right)=\frac{x^{2}-\eta^{2} y^{2}}{\left(x^{2}+\eta^{2} y^{2}\right)^{2}}, \\
\frac{\partial}{\partial y}\left(\frac{x}{x^{2}+\eta^{2} y^{2}}\right) & =-\eta \frac{2 x \eta y}{\left(x^{2}+\eta^{2} y^{2}\right)^{2}},
\end{aligned}
$$

so lautet der Ausdruck für die Polarisationsladungen $\varrho^{\prime}$ statt $(2,5)$ jetzt:

$$
\varrho^{\prime}=d_{1}{ }^{\prime} \frac{\cos 2 \Phi^{\prime}}{R^{2}}+d_{2}{ }^{\prime} \frac{\sin 2 \Phi^{\prime}}{R^{2}}
$$

mit

$$
\begin{aligned}
& d_{1}{ }^{\prime}=\frac{1}{2}(b \eta / \pi)\left(e_{1,23}^{\prime}+e_{2,13}^{\prime}\right), \\
& d_{2}{ }^{\prime}=\frac{1}{2}(b \eta / \pi)\left(e_{2,23}^{\prime}-\frac{1}{\eta^{2}} e^{\prime}{ }_{1,13}\right) .
\end{aligned}
$$

Dabei wurde gesetzt:

$R=\sqrt{X^{2}+Y^{2}}, \Phi^{\prime}=\operatorname{arctg}(Y / X)$ mit $X \equiv x, Y \equiv \eta y$.
Damit $\varrho^{\prime} \equiv 0$ und folglich auch $V \equiv 0$ ist, müssen $d_{1}{ }^{\prime}$ und $d_{2}{ }^{\prime}$ wieder gleichzeitig verschwinden.

Wir betrachten zunächst die kubischen Kristalle. Lassen wir in (A,5a) die für die Diskussion unwichtigen Vorfaktoren fort und denken uns wieder die Ausdrücke $(3,4)$ für die $e_{a, \beta}^{\prime}$, eingesetzt, so besteht der einzige Unterschied gegenüber den Gln. $(3,5)$ offensichtlich darin, daß statt des zweiten Faktors in $(3,5 \mathrm{~b})$ jetzt der Faktor auftritt:

$$
3 \cos ^{2} \alpha+3 \cos ^{2} \beta-\left(1+1 / \eta^{2}\right) .
$$

Es könnten daher allenfalls diejenigen Richtungen den Charakter einer Ausnahmerichtung verlieren, für die das Verschwinden dieses zweiten Faktors in $(3,5 \mathrm{~b})$ vorausgesetzt war, nämlich die $\langle 111\rangle$-Richtungen (Fall $\beta$ ). Da für sie aber, wie erwähnt, gerade $\eta=1$ gilt, stimmt dieser Faktor wieder mit dem zweiten Klammerfaktor in $(3,5 b)$ überein, verschwindet also nach wie vor.

Für die hexagonalen Kristalle brauchen wir wieder nur $d_{2}{ }^{\prime}$, d. h.

$$
e_{2,23}^{\prime}-\eta^{-2} e_{1,13}^{\prime}
$$

zu betrachten. Nach Einsetzen von $(3,8)$ ergibt sich gegenüber der rechten Seite von $(3,9 \mathrm{~b})$ jetzt das $\mathrm{Zu}$ satzglied:

$$
\left(1-\eta^{-2}\right) \cos \gamma e_{15}
$$

Dies verschwindet aber für die dort erhaltenen Ausnahmerichtungen ebenfalls: Für die Schraubenversetzung entlang der $c$-Achse wegen $\eta=1$, für diejenigen in der hexagonalen Basisebene wegen $\cos \gamma=0$. Damit ist die Behauptung in allen Teilen bewiesen. 